Alexandre Henrique Macchetti ${ }^{1}$

Heitor Ricardo Cosiski Marana ${ }^{2}$

\section{Densidade mamográfica como fator de risco para o câncer de mama}

\author{
Mammographic density as risk factor for breast cancer
}

$\mathrm{O}$ aspecto radiográfico da mama varia entre as mulheres conforme a sua composição por gordura, estroma e epitélio tecidual, que possuem diferentes propriedades de atenuação radiográfica. Há mais de 30 anos, John Wolfe ${ }^{1}$ descrevia a associação de mamas com um padrão mamográfico de alta densidade, ou seja, maior proporção de tecido glandular e estromal em relação ao tecido gorduroso na mama ao aumento do risco para o câncer de mama - associação atualmente confirmada por muitos estudos bem conduzidos, que justificam a análise da incorporação da densidade mamográfica aos modelos de predição de risco individual de câncer de mama, como o de Gail ${ }^{2,3}$.

A densidade mamográfica depende de muitos fatores, como número de filhos, peso corpóreo e idade, mas é independente dos níveis circulantes de hormônios sexuais na pós-menopausa ${ }^{4}$. Uma alta densidade mamográfica é mais comumente vista em mulheres jovens, enquanto a densidade diminui com a idade e apresenta grande declínio na perimenopausa ${ }^{5-7}$.

Foram propostos vários métodos para avaliação da densidade mamográfica ${ }^{8}$, mas três métodos qualitativos têm sido mais empregados. Um deles é a classificação de Wolfe$^{1}$ (N1, mama gordurosa normal; P1 e P2, ductos proeminentes ocupando menos que $25 \%$ e entre 25 e $75 \%$ da mama, respectivamente; Dy, mama displásica, com extensa região de densidade mamográfica). Outra, mais recente e amplamente utilizada na prática clínica, é a classificação do Breast Imaging Reporting and Data System (BI-RADS) ${ }^{9,10}$ do Colégio Americano de Radiologia. Outras classificações empregam os percentuais de densidade, como a de Boyd ${ }^{7}$.

Em recente meta-análise, em que se empregou a dicotomização da classificação de Wolfe $(\mathrm{N} 1+\mathrm{P} 1$ versus $\mathrm{P} 2+\mathrm{Dy})$, demonstrou-se que a incidência e a prevalência de câncer de mama foram, respectivamente, 1,86 e 1,44 vezes maiores no grupo de alto risco e, mesmo se comparados os padrões P1, P2 e Dy ao N1, observa-se o risco relativo de câncer de mama, respectivamente, de 1,76, 3,05 e 3,98 em estudos de incidência para o câncer de mama².

Correspondência:

Heitor Ricardo Cosiski Marano Departamento de Ginecologia e Obstetrícia do Hospital das Clínicas da Faculdade de Medicina de Ribeirão Preto da Universidade de São Paulo Avenida Bandeirantes, 3.900, 8 andar - Campus Universitário CEP $14049-900$ - Ribeirão Preto/SP Fone: (16) 3602-2583 Fax: (16) 3633-0946 E-mail: ahmacchetti@yahoo.com.br
Médico Assistente do Centro Integrado de Diagnóstico por Imagem - Cidi - São Carlos (SP), Brasil; Pós-graduando do Departamento de Ginecologia e Obstetrícia da Faculdade de Medicina de Ribeirão Preto da Universidade de São Paulo - FMRP-USP - Ribeirão Preto (SP), Brasil.

${ }_{2}$ Médico Assistente do Departamento de Ginecologia e Obstetrícia da Faculdade de Medicina de Ribeirão Preto da Universidade de São Paulo - FMRP-USP - Ribeirão Preto (SP), Brasil. 
A classificação BI-RADS, inicialmente composta por quatro categorias descritivas de composição mamária (quase totalmente gordurosa, densidade fibroglandular, heterogeneamente densa e extremamente densa), em sua quarta edição, associou a abordagem quantitativa com a divisão em quartis da composição percentual por tecido glandular na mama. Embora esta última versão implique alguma subjetividade, ela apresenta a vantagem de ser rotineiramente empregada em laudos mamográficos, o que facilita sua aplicação em estudos epidemiológicos ${ }^{9,10}$. Comparadas as mamas categorizadas como extremamente densas com mamas lipossubstituídas, observou-se que há um aumento do risco relativo de quatro vezes para desenvolvimento do câncer de mama entre as primeiras ${ }^{11}$.

Entretanto, a variabilidade e a reprodutibilidade permanecem como os maiores problemas das classificações das mamas em padrões de parênquima. Estudo de Nicholson et al. ${ }^{12}$ mostrou que os padrões BI-RADS, com mamas quase totalmente compostas por tecido adiposo ou as extremamente densas, identificados por radiologistas, correlacionam-se muito bem com os percentuais de densidade obtidos com método computadorizado, enquanto as demais categorias, intermediárias, tiveram variação ampla, o que dificultaria sua utilização em estudos para cálculo de risco.

Estudo de Boyd et al. ${ }^{13}$ confirma a importância de uma determinação precisa da densidade, pois observaram o aumento de $2 \%$ no risco relativo para cada aumento de $1 \%$ no percentual de densidade mamográfica. Assim, a utilização de métodos quantitativos computadorizados gera melhores aferições da densidade, com melhor reprodutibilidade ${ }^{14}$, mesmo quando se empreguem programas de auxílio ao diagnóstico de lesões ${ }^{15,16}$ no processo de análise, em vez de programas especializados. No entanto, estes métodos assistidos por computador têm, atualmente, aplicação limitada na prática diária, devido ao tempo dispendido em cada análise.

No estudo publicado em 2007 por Boyd et al. ${ }^{7}$ no New England Journal of Medicine, foi analisada a associação do percentual de densidade na mamografia inicial com o risco de câncer de mama, de acordo com o método de detecção do câncer, tempo desde o início do rastreamento e idade. Neste estudo caso-controle, realizou-se ajuste por idade, paridade, idade da menarca e menopausa, índice de massa corporal (IMC) e existência de parentes de primeiro grau com câncer de mama, entre outros. Os resultados mostram que, comparadas com mulheres nas quais as áreas densas ocupam superfície menor que $10 \%$ da mamografia, as mulheres com $75 \%$ ou mais têm um aumento de risco de câncer de mama $(O d d$ s Ratio $(\mathrm{OR})=4,7$; intervalo de confiança (IC) de $95 \%=3,0$ a 7,4), enquanto detectado por rastreamento $(\mathrm{OR}=3,5 ; \mathrm{IC} 95 \%=2,0$ a 6,2$)$ ou menos que 12 meses após exame de rastreamento negativo (OR=17,8; IC95\%=4,8 a 65,9).

Estabelecendo-se a associação inequívoca da densidade com o risco de câncer de mama, surge a preocupação com as evidências de que terapias hormonais poderiam alterar a densidade mamográfica e, talvez, o risco de câncer de mama. Nos estudos de Freedman et al. ${ }^{17}$ e McTiernan et al. ${ }^{18}$ - dois trials randomizados, placebo-controlados e duplo-cegos -, foram utilizados métodos quantitativos para a avaliação do efeito da terapia hormonal sobre a densidade mamográfica. Ambos mostram claramente que a terapia estrogênica foi capaz de aumentar a densidade mamográfica percentual em um curto espaço de tempo (dois anos), em 1,2 e 4,9\%, respectivamente, enquanto no grupo controle ocorreu diminuição percentual de 1,3 e $0,8 \%$ na densidade mamográfica. Outros recentes estudos prospectivos observacionais, também utilizando métodos de avaliação quantitativa, encontraram evidências de que o uso da terapia estrogênica diminui ou impede o declínio da densidade mamográfica normalmente observada com o envelhecimento ${ }^{5,19,20}$, o que poderia se traduzir com o passar dos anos em um aumento do risco de câncer de mama.

A alteração da densidade mamográfica pela terapia hormonal com estrógenos e progestágenos aumenta o interesse por drogas como o raloxifeno. Este é um modulador seletivo do receptor de estrógeno (SERM) de segunda geração, inicialmente aprovado pela Food and Drugs Administration (FDA) para prevenção e tratamento da osteoporose, mas que mostrou efeitos antiestrogênicos na mama ${ }^{21}$, indicando ser tão efetivo quanto o tamoxifeno para reduzir o risco de carcinoma invasivo de mama ${ }^{22}$, tendo, inclusive, o efeito de reduzir a vasculatura em tumores mamários receptor de estrógenos positivos ${ }^{21}$.

O efeito do raloxifeno sobre a densidade mamográfica avaliado por Silvério et al. ${ }^{23}$ é apresentado neste fascículo da Revista Brasileira de Ginecologia e Obstetrícia (RBGO). O estudo prospectivo destes autores, utilizando a classificação BI-RADS e a técnica assistida por computador, mostrou a manutenção da densidade mamográfica após uso de raloxifeno, o que confirma os dados da literatura ${ }^{24}$. 
A diminuição da incidência de câncer de mama com a utilização profilática do tamoxifeno poderia ser explicada parcialmente pela capacidade de modificar a proporção relativa dos diversos compartimentos de parênquima mamário, com resultado sobre o amplamente conhecido efeito de diminuir a densidade mamográfica ${ }^{25}$. Esta correlação permite supor que o monitoramento desta densidade poderia vir a ser um item indicativo de benefício com uma intervenção hormonal, antiestrogênica ou não. Uma destas propostas pode ser a associação do tamoxifeno em baixa dose ao esquema de terapia hormonal em uso. Um estudo recente $^{26}$ pôde mostrar que esta associação modulou favoravelmente marcadores de carcinogênese mamária e risco cardiovascular sem aumento de proliferação endometrial ou sintomas menopausais.

\section{Referências}

1. Wolfe JN. Risk for breast cancer development determined by mammographic parenchymal pattern. Cancer. 1976;37(5):2486-92.

2. McCormack VA, Santos Silva I. Breast density and parenchymal patterns as markers of a breast cancer risk: a meta-analysis. Cancer Epidemiol Biomarkers Prev. 2006;15(6): 11 159-69.

3. Chen J, Pee D, Ayyagari R, Graubard B, Schairer C, Byrne C, et al. Projecting absolute invasive breast cancer risk in white women with a model that includes mammographic density. J Natl Cancer Inst. 2006;98(17):1215-26.

4. Verheus M, Peeters PH, van Noord PA, van der Schouw YT, Grobbee DE, van Gils CH. No relationship between circulating levels of sex steroids and mammographic breast density: the Prospect-EPIC cohort. Breast Cancer Res. 2007;9(4):R53.

5. Maskarinec G, Pagano I, Lurie G, Kolonel LN. A longitudinal investigation of mammographic density: the multiethnic cohort. Cancer Epidemiol Biomarkers Prev. 2006;15(4):732-9.

6. Boyd NF, Rommens JM, Vogt K, Lee V, Hopper JL, Yaffe M, et al. Mammographic breast density as an intermediate phenotype for breast cancer. Lancet Oncol. 2005;6(10):798-808.

7. Boyd NF, Guo H, Martin L, Sun L, Stone J, Fishell E, et al. Mammographic density and the risk and detection of breast cancer. N Engl J Med. 2007;356(3):227-36.

8. Macchetti AH, Marana HRC, Andrade JM, Spina L, Bighetti S. Alterações mamográficas relacionadas à terapia de reposição hormonal. Femina. $2001 ; 29(6): 365-9$.

9. American College of Radiology. Breast Imaging Reporting and Data System (BI-RADS). $4^{\text {th }}$ ed. Reston: American College of Radiology; 2003.

10. Balleyguier C, Ayadi S, Van Nguyen K, Vanel D, Dromain C, Sigal R. BIRADS classification in mammography. Eur J Radiol. 2007;61(2): 192-4.

11. Vacek PM, Geller BM. A prospective study of breast cancer risk using routine mammographic breast density measurements. Cancer Epidemiol Biomarkers Prev. 2004;13(5):715-22.

12. Nicholson BT, LoRusso AP, Smolkin M, Bovbjerg VE, Petroni GR, Harvey JA. Accuracy of Assigned BI-RADS breast density category definitions. Acad Radiol. 2006;13(9):1 143-9.

13. Boyd NF, Greenberg C, Lockwood G, Little L, Martin L, Byng J, et al. Effects at two years of a low-fat, high-carbohydrate diet on radiologic features of the breast: results from a randomized trial. Canadian Diet and Breast Cancer Prevention Study Group. J Natl Cancer Inst. 1997;89(7):488-96.

14. Martin KE, Helvie MA, Zhou C, Roubidoux MA, Bailey JE, Paramagul C, et al. Mammographic density measured with quantitative computeraided method: comparison with radiologists' estimates and BI-RADS categories. Radiology. 2006;240(3):656-65.

15. Kinoshita SK, de Azevedo-Marques PM, Pereira RR Jr, Rodrigues JA, Rangayyan RM. Content-based retrieval of mammograms using visual features related to breast density patterns. J Digit Imaging. 2007;20(2):172-90.

16. Kinoshita SK, Marques PMA, Slaets AF, Marana HRC, Ferrari RJ. Characterization of breast masses using texture and features. In: Doi K, McMahon H, Giger ML, Hoffmann KR, editors. Computer-aided diagnosis in medical imaging. Amsterdam: Elsevier; 1999. p. $265-70$.

17. Freedman M, San Martin J, O'Gorman J, Eckert S, Lippman ME, Lo SC et al. Digitized mammography: a clinical trial of postmenopausal women randomly assigned to receive raloxifene, estrogen, or placebo. J Natl Cancer Inst. 2001;93(1):51-6.

18. McTiernan A, Martin CF, Peck JD, Aragaki AK, Chlebowski RT, Pisano ED, et al. Estrogen-plus-progestin use and mammographic density in postmenopausal women: women's health initiative randomized trial. J Natl Cancer Inst. 2005;97(18):1366-76.

19. Van Duijnhoven FJ, Peeters PH, Warren RM, Bingham SA, van Noord PA, Monninkhof EM, et al. Postmenopausal hormone therapy and changes in mammographic density. J Clin Oncol. 2007;25(1 1):1323-8.

20. da Silva BB, Lopes IM, Gebrim LH. Effects of raloxifene on normal breast tissue from premenopausal women. Breast Cancer Res Treat. 2006;95(2):99-103.

21. Vogel VG, Costantino JP, Wickerham DL, Cronin WM, Cecchini RS, Atkins JN et al. Effects of tamoxifen vs raloxifene on the risk of developing invasive breast cancer and other disease outcomes: the NSABP Study of Tamoxifen and Raloxifene (STAR) P-2 trial. JAMA. 2006;295(23):2727-41. 
22. Lopes-Costa PV, Santos AR, Pires CG, Pereira Filho JD, Silva BB. Efeitos do raloxifeno sobre a angiogênese do carcinoma de mama de mulheres menopausadas. Rev Bras Ginecol Obstet. 2007;29(7):335-9.

23. Silvério CD, Nahas-Neto J, Nahas EAP, Guazeelli MMO, Gomes MA, Dias R. Efeito do raloxifeno sobre a densidade mamográfica em mulheres na pós-menopausa. Rev Bras Ginecol Obstet. 2007;29(10):paginação?

24. Lasco A, Gaudio A, Morini E, Morabito N, Nicita-Mauro C, Catalano A, et al. Effect of long-term treatment with raloxifene on mammary density in postmenopausal women. Menopause. 2006;13(5):787-92.

25. Cuzick J, Warwick J, Pinney E, Warren RM, Duffy SW. Tamoxifen and breast density in women at increased risk of breast cancer. J Natl Cancer Inst. 2004;96(8):621-8.

26. Decensi A, Gandini S, Serrano D, Cazzaniga M, Pizzamiglio M, Maffini F, et al. Randomized dose-ranging trial of tamoxifen at low doses in hormone replacement therapy users. J Clin Oncol. 2007;25(27):4201-9. 